\title{
Diagnostic test evaluation of a nasal flow monitor for obstructive sleep apnea detection in sleep apnea research
}

\author{
KEITH K. H. WoNG \\ University of Sydney, Sydney, New South Wales, Australia \\ David Jankelson and Adrian ReID \\ St. Vincent's Hospital, Darlinghurst, New South Wales, Australia \\ GunNar Unger and George Dungan \\ University of Sydney, Sydney, New South Wales, Australia \\ JAN A. HEDNER \\ Sahlgrenska University Hospital, Göteborg, Sweden \\ AND \\ RONALD R. GRUNSTEIN \\ University of Sydney, Sydney, New South Wales, Australia
}

\begin{abstract}
In this diagnostic test evaluation of a nasal flow monitoring device for obstructive sleep apnea (OSA), 34 patients referred for polysomnography were studied at home for three consecutive nights with the monitor. The mean age of subjects $( \pm S D)$ was $41.9 \pm 10.3$ years, and their mean apnea-hypopnea index (AHI) was 31.5 \pm 27.2 . The difference between the average AHI from three nights at home on the monitor and the polysomnogram (PSG) result was $1.8 \pm 17.1$. The area under the receiver operating characteristic curve (AUC) for PSG AHI $\geq 10$ was .96 . With a threshold AHI of 18 on the flow monitor, sensitivity was .92 , specificity .86, positive predictive value .96 , and negative predictive value .75. For detecting severe OSA (AHI $\geq 30$ ), the AUC was .85 . With knowledge of appropriate thresholds and the pretest risk of OSA, the flow monitor can be used to detect or exclude OSA for sleep-related research, as well as to identify severe cases needing priority for further evaluation.
\end{abstract}

\section{Background}

Sleep is a ubiquitous phenomenon that, although a biological imperative, is both associated with and affected by various pathologies, including obstructive sleep apnea, a common sleep disorder affecting $9 \%-24 \%$ of an adult population (Young et al., 1993), resulting in significant neurocognitive effects (Engleman \& Joffe, 1999).

Behavioral research often must take a subject's sleep status into consideration when applying testing or interpreting the results. This ability to quantify disordered breathing is essential as part of inclusion or exclusion criteria for behavioral research related to sleep. Such information is essential to many trials evaluating the effects of sleep deprivation, sleep loss, shift work, metabolism, pharmacotherapy, and other sleep pathologies; all of these factors may be affected by sleep-disordered breathing. Symptoms of obstructive sleep apnea (OSA), such as snoring, choking in sleep, and excessive daytime sleepiness, are common and nonspecific. Although
$28 \%$ of patients presenting themselves for primary care report snoring more than three times per week (Netzer, Stoohs, Netzer, Clark, \& Strohl, 1999), OSA is present in only $66 \%$ of male and $19 \%$ of female habitual snorers (Young et al., 1993). Moreover, insomnia symptoms are also frequently found in patients with OSA, and it may be important to differentiate these conditions in behavioral research (Gooneratne et al., 2006).

The in-laboratory polysomnogram (PSG) is the recommended test to confirm the diagnosis of OSA. This test is resource-intensive to administer, and there is debate about the effect of night-to-night variability on the diagnosis of OSA when testing is only conducted on a single night (Le Bon et al., 2000). Behavioral researchers may not have easy access, however, to full in-laboratory polysomnography for purposes of screening subjects for investigation. Several alternative diagnostic devices have been evaluated in the diagnosis of OSA, but these were developed mainly with the aim of reducing cost (Flemons et al., 2003). 
Diagnostic devices for OSA have been broadly classified into four types. Type 1 is the standard in-laboratory PSG with a minimum of seven channels, and Type 2 is a comprehensive portable PSG with unattended measurement of all channels used in a standard PSG. Type 3 devices contain a minimum of four recording channels, including respiratory movement, respiratory airflow, heart rate, and oxygen saturation, and Type 4 devices provide single- or dual-channel recording (Flemons et al., 2003). These devices represent descending levels of technical complexity.

In this study, we determined the diagnostic accuracy of a single-channel nasal flow monitor in the evaluation of OSA in patients presenting themselves at a sleep center. We aimed primarily to assess the ability of such a monitor to exclude sleep apnea in those who do not have the condition, and secondarily to assess its ability to detect those with severe OSA. Furthermore, we examined the additional value of a questionnaire instrument and demographic variables in the diagnosis of OSA.

\section{METHOD}

Consecutively presenting patients who were referred to the sleep center with suspected sleep-disordered breathing were recruited if they were due to have diagnostic polysomnography, were 18 to 55 years old, gave written informed consent, and were able to comply with the study procedures. No specific exclusion criteria were defined. The sleep center comprises a two-bed laboratory within a metropolitan teaching hospital (St. Vincent's Hospital, Sydney, New South Wales, Australia). The study was approved by the institutional ethics committee of St. Vincent's Hospital (Reference H03/119).

\section{Subjects}

Thirty-four subjects were recruited over a 3-month period. One subject was female. Their age (mean $\pm S D$ ) was $41.9 \pm 10.3$ years, mean body mass index (BMI) was $30.2 \pm 5.4 \mathrm{~kg} / \mathrm{m}^{2}$, and their mean Epworth sleepiness scale score was 11.9 44.7. Mean PSG apneahypopnea index (PSG AHI) was $31.5 \pm 27.2 / \mathrm{h}$ (range 0-100). Twentysix patients $(79 \%)$ had obstructive sleep apnea (PSG AHI $\geq 10$ ), and $13(39 \%)$ had severe OSA (PSG AHI $\geq 30$ ).

One subject (male, age 57 years) withdrew from the study before having his PSG. Another 2 subjects (6\%) had inadequate data for all three nights at home on the flow monitor, and 1 other $(3 \%)$ had inadequate data for the first night at home on the flow monitor.

\section{Reference Standard: In-Laboratory Polysomnography}

The in-laboratory PSG was acquired using commercially available equipment (Alice 4; Respironics, Murrysville, PA). The following signals formed the montage: $\mathrm{C} 4 / \mathrm{A} 1, \mathrm{C} 3 / \mathrm{A} 2, \mathrm{O} 2 / \mathrm{A} 1$, and $\mathrm{O} 1 / \mathrm{A} 2$ electroencephalogram; right and left electrooculogram; submental electromyogram; thoracic and abdominal piezoelectric respiratory movement sensors; oxygen saturation; nasal-oral thermistor; nasal pressure via cannulae; tibialis anterior electromyogram; body position; sound (snoring); and single-lead electrocardiogram. The subjects were attended during the entire recording period by sleep laboratory staff.

The polysomnographic records were scored manually using standard techniques (American Academy of Sleep Medicine Task Force, 1999; Rechtschaffen \& Kales, 1968) by a single scorer who was blinded to the results of the flow monitor. Sleep was staged in 30-sec epochs. Respiratory events were scored using 5-min epoch displays. An apnea was defined as a cessation of airflow on the nasal pressure channel for at least $10 \mathrm{sec}$. A hypopnea was defined as a period of reduction in nasal airflow for at least $10 \mathrm{sec}$, accompanied by an electroencephalographic arousal (Sleep Disorders Atlas Task Force of the American Sleep Disorders Association, 1992) or a 3\% oxygen desaturation. The apneahypopnea index (AHI) is defined as the total number of apneas and hypopneas per hour of sleep. Subjects were diagnosed with OSA on the basis of an $A H I \geq 10$. Severe OSA was defined as an $A H I \geq 30$.

\section{Nasal Airflow Monitor}

The nasal airflow monitor (see Figure 1; Flow Wizard, DiagnoselT, Sydney, New South Wales, Australia) consists of both a recording device and analysis software. The recorder (dimensions $11.5 \times 7 \times$ $4.2 \mathrm{~cm}, 148 \mathrm{~g}$ including batteries) employs a pressure transducer, analog-to-digital conversion, and storage hardware to record the masal airflow collected by means of standard nasal oxygen cannulae (Unger, Hedner, Grunstein, \& Williams, 2003). The device was designed around a "distributed" method for management of patient diagnostic information. This model allows the data to be held in a central server and offers all interested parties (e.g., referring physicians or specialists) access to the data via a thin-client approach.

Patients were instructed to wear the nasal cannulae of the flow monitor upon retiring to bed and to press a button on the device that triggered the recording of the signal for $9 \mathrm{~h}$. The recording was performed for three consecutive nights at home before subjects returned the device for downloading.

On the night of the in-laboratory PSG, the flow from the patient was simultaneously monitored by both the flow-monitoring recorder and the PSG during the recording by means of a three-way connector.

Data were sampled at 25 samples/sec, filtered, amplified, and digitized within the recorder, and subsequently stored in nonvolatile memory capable of recording a total of $27 \mathrm{~h}$ of monitoring data. The recordings were downloaded from the recorder to a personal computer by a research assistant blinded to the results of the polysomnography. The software provided with the device employed an algorithm to analyze characteristics including the amplitude and stability of the flow/pressure curve in order to distinguish snoring and respiratory events from normal breathing.

The software evaluated the flow signal for periods of the recording in which data quality was inadequate or absent (commonly a secondary effect of movement artifact or cannula displacement). These periods are excluded from analysis. Individual recording nights with less than $4 \mathrm{~h}$ of adequate data were excluded from analysis. Signals were adjusted for DC drift. The detection of apnea and hypopnea was achieved by using amplitude thresholds that were set using a 20-breath weighted moving averages of "nominal" airflow as recorded on the Flow Wizard monitor. Apneas were identified as episodes in which the absolute flow signal dropped below a near-zero threshold value for at least $10 \mathrm{sec}$. Absence of flow for $120 \mathrm{sec}$ was deemed to be unlikely to be associated with an apnea event, and the data were excluded. Hypopneas were defined using similar detection methodologies, with an event threshold of $50 \%$ of the nominal maximum flow occurring for at least $10 \mathrm{sec}$.

Periods of flow limitation were evaluated on a breath-by-breath basis using curve matching algorithms for the inspiratory limb of the ventilation. Snoring is also monitored, sampling the pressure

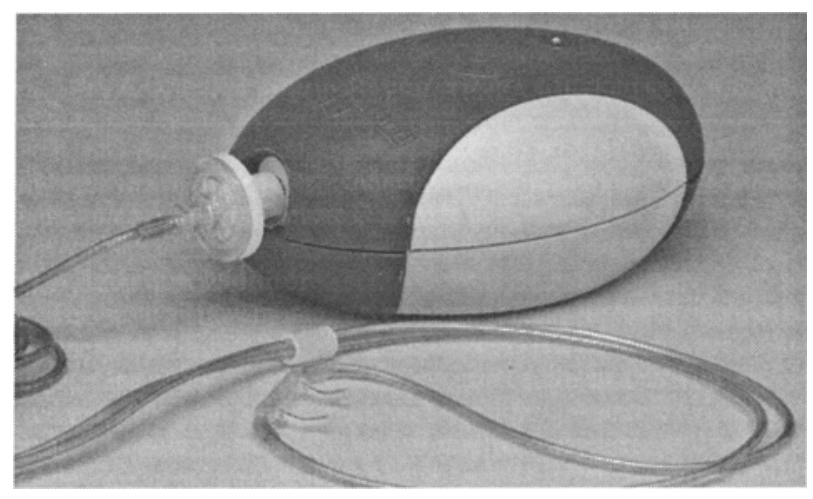

Figure 1. Illustration of the Flow Wizard device. Nasal pressure cannula are attached to the port on the left side of the pictured device. 
transducer at 2,000 samples/sec. These data were digitized and range values were stored and associated with each breath. The amount of snoring was determined by the high-frequency maximum and minimum values consistently meeting a preset limit.

Apneas and hypopneas were scored automatically, and an apneahypopnea index for this device (FW AHI) was calculated as a ratio of the total number of apnea and hypopnea events to the hours of recorded data of adequate quality (quality recording). The AHI derived for the three nights at home (FW $\mathrm{AHI}_{1-3}$ ) was calculated from the average of the included nights.

\section{Procedure}

Subjects consenting to the study were seen in the sleep center prior to the night of their PSG. They had their demographic details collected, their height and weight measured, and they were asked to complete a questionnaire of symptoms of sleep apnea comprising a validated predictive algorithm for sleep apnea diagnosis (the multivariable apnea prediction index, or MAPI; Maislin et al., 1995). They were also asked to complete the Epworth sleepiness scale (Johns, 1991). The subjects were instructed to use the flow monitor for three consecutive nights at home and to complete a sleep diary for the same three nights. On a separate visit to the sleep center, they underwent an in-laboratory overnight PSG (fourth night) that was done with simultaneous flow monitor recording.

\section{Statistical Analysis}

Bland-Altman plots were used to compare the average AHI derived from the flow monitor during the three nights at home (FW $\mathrm{AHI}_{1-3}$ ) with the AHI derived by the gold standard polysomnogram (PSG AHI) (Bland \& Altman, 1986).

To assess the utility of the flow monitor for excluding significant OSA, the diagnosis of OSA was defined by a PSG AHI $\geq 10$. Receiver operating characteristic (ROC) curves were used to explore the relationship between sensitivity and specificity, and the area under the curve was calculated. Diagnostic performance indicesthe sensitivity, specificity, likelihood ratios, and predictive valueswere determined at thresholds with high sensitivity, high specificity, and the highest diagnostic odds ratio (ratio of positive to negative likelihood ratios), and at the point on the ROC curve closest to the upper left corner. The likelihood ratio indicates the extent to which a test will raise or lower the pretest odds of the diagnosis (OSA) being present. Likelihood ratios of 5-10 and 0.1-0.2 generate moderate shifts in pretest to posttest odds, whereas values $>10$ or $<0.1$ generate large and often conclusive changes from pretest to posttest odds (Jaeschke, Guyatt, \& Sackett, 1994). To assess the utility of the flow monitor to detect severe OSA, the analysis was referenced to severe OSA (PSG AHI $\geq 30$ ). ROC curves and diagnostic performance indices were reported as above.

Data from the flow monitor (Night 4) and polysomnogram recorded simultaneously in the sleep laboratory were examined to evaluate the performance of the flow monitor under ideal circumstances-that is, with possible sources of variation, such as internight variability in OSA severity, eliminated. A Bland-Altman (1986) plot and the area under the ROC curve were examined.

The ROC analyses were compared with the data obtained from the flow monitor on the first night at home and with the questionnaire predictive tool. Logistic regression modeling was employed to explore the additive value of the MAPI, Epworth sleepiness scale score, and demographic variables in the diagnostic accuracy of the flow monitor. A two-sided significance level of 5\% was applied. These analyses were performed using $R$ software (www.r-project.org).

\section{RESULTS}

\section{Bland-Altman Limits of Agreement Analysis}

Figure 2 depicts the Bland-Altman (1986) plot showing the difference in AHI derived by the average of the

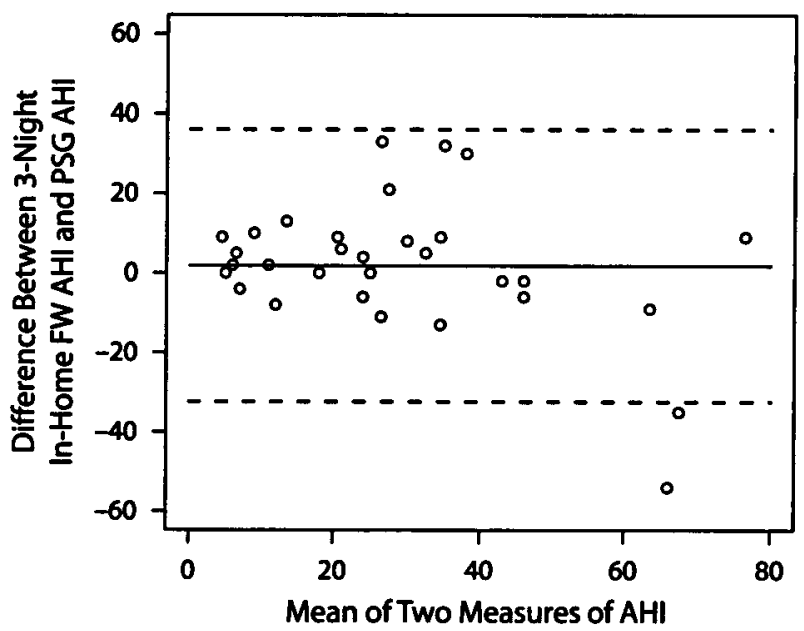

Figure 2. Bland and Altman (1986) plot comparing the apneahypopnea index (AHI) derived from three nights on flow monitor (FW) with AHI scored in an in-laboratory polysomnogram (PSG).

three nights at home on the flow monitor $\left(\mathrm{FW} \mathrm{AHI}_{1-3}\right.$ ), as compared with the gold standard single-night PSG AHI $(N=31$ subjects). The mean difference (bias) between the two measurements was $1.8 \pm 17.1 / \mathrm{h}$, with greater differences seen with high AHI. Limits of agreement were calculated as $2 S D$ s from the mean difference. The lower limit of agreement was $-32.4 / \mathrm{h}$, and the upper limit was $36.0 / \mathrm{h}$ (dashed lines in figure). Limits of agreement applicable to the population, including the widest extent of the $95 \%$ confidence intervals for the upper and lower limits of agreement, were -43.1 to 46.8 .

\section{Ruling Out Obstructive Sleep Apnea}

We assessed the characteristics of the flow monitor in ruling out OSA (PSG AHI $\geq 10$ ). Figure 3 (solid line) shows the ROC curves for the three-night home recording of the flow monitor. The area under the curve (AUC) was .96. If data obtained only from the first night on the flow monitor had been used, the area under the curve (dashed line) would be .92 .

Table 1 includes sensitivity, specificity, likelihood ratios, and predictive values at various cut points. A threshold of 18 events $/ \mathrm{h}$ on the flow monitor yielded the highest diagnostic odds ratio (ratio of positive to negative likelihood ratios), where the sensitivity was .92 and specificity .86 .

\section{Identifying Severe OSA}

Figure 4 (solid line) shows the ROC curve for the FW $\mathrm{AHI}_{1-3}$ in detecting severe OSA (PSG AHI $\geq 30$ ). The AUC was .85. The test characteristics are described in Table 2. A threshold of $\mathbf{2 8}$ on the flow monitor yielded a sensitivity of .91 and specificity of .75 in detecting severe OSA. If only the data from the first night at home on the flow monitor had been considered, the AUC (Figure 4, dashed line) would be .89 . 


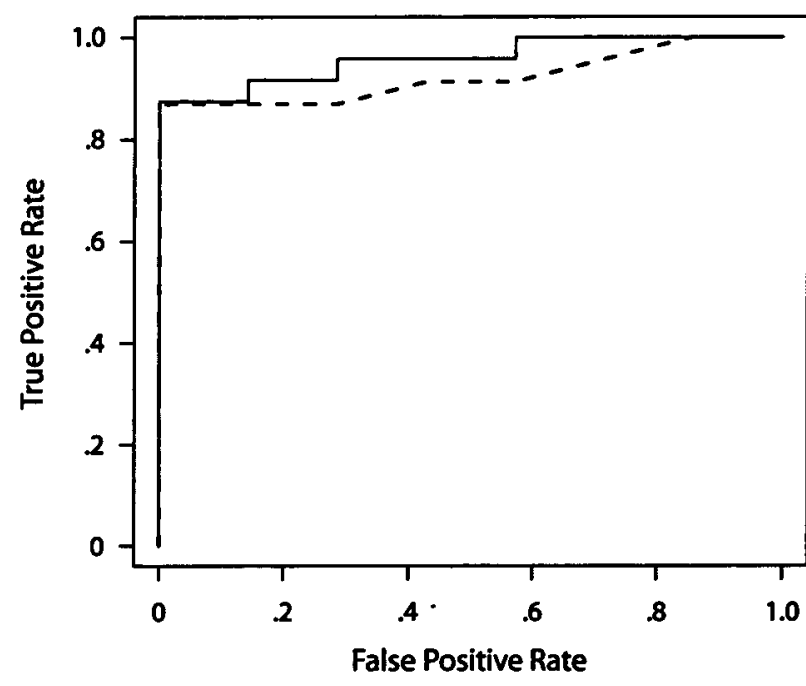

Figure 3. Recelver operating characteristic curve for the flow monitor applied at home with respect to the diagnosis of sleep apnea (PSG AHI $\geq 10$ ). The solid line represents data from the average of all three nights at home, and the dashed line represents data from only the first of the three nights.

\section{Single-Night Data and Recording \\ Simultaneously With PSG}

Subjects also underwent a flow monitor recording on the night of their PSG. Figure 5 depicts the Bland-Altman (1986) plot showing the difference between the AHI measured on the flow monitor and the simultaneously measured PSG AHI ( $N=31$ subjects). The mean difference (bias) between the two measurements was $7.1 \pm 11.1 / \mathrm{h}$. The lower limit of agreement was $-14.8 / \mathrm{h}$, and the upper limit was $29.0 / \mathrm{h}$ (dashed lines in figure). Limits of agreement applicable to the population, including the widest extent of the $95 \%$ confidence intervals for the upper and lower limits of agreement, were -21.7 to 35.9 .

With the same optimal threshold of 18 events/h determined during the home recordings of the flow monitor, the sensitivity was .96 , specificity .71 , positive likelihood ratio 3.37, and negative likelihood ratio 0.05 for detecting OSA.

The AUC for the single in-laboratory night on the flow monitor with respect to the presence of OSA (PSG $\mathrm{AHI} \geq 10$ ) was .95 . The areas under the ROC curve for the three nights on the flow monitor at home, when analyzed separately, were $.92, .96$, and .94 with respect to PSG-defined OSA.

\section{Addition of Other Predictive Factors to the Flow Monitor to Improve Diagnostic Accuracy}

The MAPI calculation utilized information from questionnaire responses regarding three breathing symptoms of OSA (snoring, breathing stops, and choking), BMI, gender, and age to yield a value from 0 to 1 , in order to estimate the probability of OSA (Maislin et al., 1995). Univariate logistic regression considering the individual variables comprising the MAPI, the overall MAPI score, and the score on the Epworth sleepiness scale show the flowmonitor-derived three-night average $\mathrm{AHI}\left(\mathrm{FW} \mathrm{AHI}_{1-3}\right)$ to be the strongest predictor of OSA (i.e., PSG AHI $\geq 10$ ) ( $p=.00007)$, with the MAPI probability $(p=.08)$ being possibly significant. With respect to the MAPI, the AUC for detecting a PSG AHI $\geq 10$ was .68 , significantly lower than that obtained from the three nights on the flow monitor $(p=.008)$. Gender was not included in the analyses, since only 1 subject was female. Multivariate analyses did not show MAPI probability to be an independent predictor $(p=.26)$ after the flow monitor measure had been accounted for. The AUC for combined MAPI probability and $\mathrm{FW} \mathrm{AHI}_{1-3}$, as measured by the $c$ statistic, was .96 . This was no higher than with the $\mathrm{FW} \mathrm{AHI} \mathrm{AH}_{1-3}$ alone.

\section{DISCUSSION}

We have demonstrated that a simple, single-channel nasal flow measuring device used at home showed good accuracy in detecting the presence of obstructive sleep apnea. Such monitoring may have utility in sleep-related behavioral research. The study recruited consecutively presenting subjects with a wide spectrum of disease severity, the reference test was scored independently of the test being evaluated, and recording of the disease over three nights at home was used to minimize the problems of night-to-night variability and data loss.

Wide limits of agreement between the flow monitor and the PSG were observed in our study. These are comparable to those reported with other flow-based single-channel monitors, and they appear no worse than what has been reported with home polysomnography with Type 2 devices, which bear the closest resemblance to an in-laboratory PSG (Gagnadoux, Pelletier-Fleury, Philippe, Rakotonanahary, \& Fleury, 2002; Portier et al., 2000). Nevertheless, the wide limits of agreement support the consensus that single-channel devices do not perfectly replicate the scoring from a more complex polysomnographic recording

Table 1

Diagnostic Test Characteristics of the Flow Monitor (FW) Used at Home for Three Nights in Detecting Obstructive Sleep Apnea

\begin{tabular}{ccccccc}
\hline $\begin{array}{c}\text { Threshold for } \\
\text { FW AHI }\end{array}$ & Sensitivity & Specificity & $\begin{array}{c}\text { Positive } \\
\text { Likelihood Ratio }\end{array}$ & $\begin{array}{c}\text { Negative } \\
\text { Likelihood Ratio }\end{array}$ & $\begin{array}{c}\text { Positive } \\
\text { Predictive Value }\end{array}$ & $\begin{array}{c}\text { Negative } \\
\text { Predictive Value }\end{array}$ \\
\hline $21^{*}$ & .88 & 1.00 & - & 0.12 & 1.00 & .70 \\
$18^{* *}$ & .92 & .86 & 6.4 & 0.10 & .96 & .75 \\
12 & .96 & .71 & 3.4 & 0.058 & .92 & .83 \\
8 & 1.00 & .43 & 1.75 & 0.00 & .86 & 1.00 \\
\hline
\end{tabular}

Note-Sensitivity, specificity, positive likelihood ratio, negative likelihood ratio, positive predictive value, and negative predictive value for detecting polysomnogram apnea-hypopnea index $(\mathrm{AHI}) \geq 10$. "Point with the lowest distance from the upper left corner of the receiver operating characteristic curve. "*Point with the highest diagnostic odds ratio. 


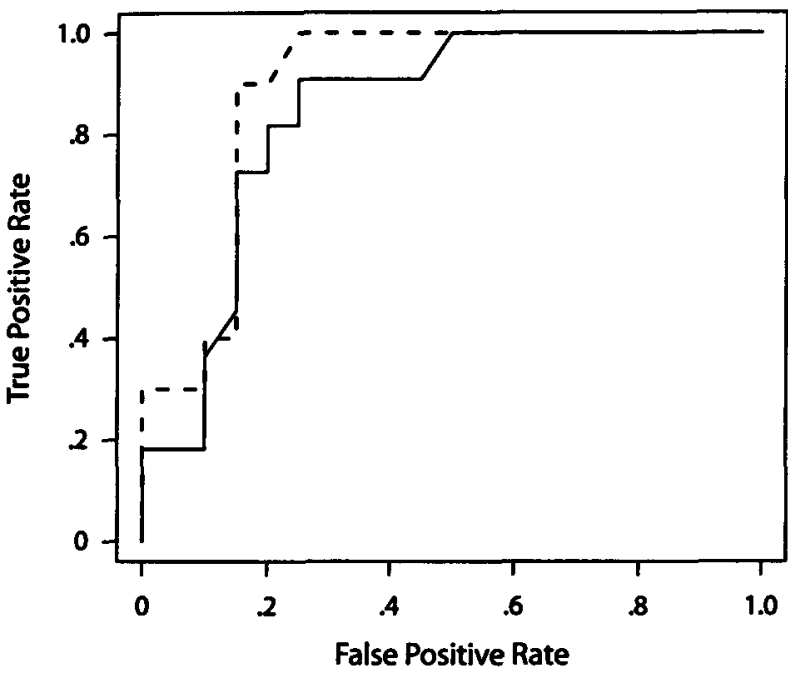

Figure 4. Recelver operating characteristic curve for the flow monitor applied at home with respect to the diagnosis of severe sleep apnea (PSG AHI $\geq 30$ ). The solid line represents data from the average of all three nights at home, and the dashed line represents data from only the first of the three nights.

(Flemons et al., 2003). Nevertheless, with clinical assessment and the use of appropriately chosen thresholds, such devices can be used to include or exclude patients for research protocols.

With the aim of excluding the presence of sleep apnea, a test with high sensitivity would be desirable. A potential subject recruited from the community (population prevalence of OSA estimated at 9\%-24\%; Young et al., 1993) who had a flow monitor AHI of less than 18 events $/ \mathrm{h}$ (negative likelihood ratio 0.10 ) would have a probability of OSA of $3 \%$ or less. Such characteristics could have application in removing patients from research protocols in which sleep apnea is an exclusionary criterion.

For other research protocols in which sleep apnea is part of the inclusion criteria, it is important to be able to screen patients for apnea, minimizing the need for the use of costly and resource-intensive polysomnography. A threshold of $28 / \mathrm{h}$ on the flow monitor provided a balance between sensitivity and specificity, as indicated by a maximal diagnostic odds ratio and the position of the threshold on the upper left inflection of the ROC curve. Those testing positive on the flow monitor with a threshold of $28 / \mathrm{h}$ had a $67 \%(10 / 15)$ rate of truly having severe sleep apnea, and only $6 \%(1 / 16)$ of patients with severe sleep apnea were not detected early. The choice of diagnostic threshold depends on many factors, including the purpose for which the test is to be applied, population factors such as the background risk of sleep apnea, cost and availability of the in-laboratory polysomnography, and the value placed on the need for early diagnosis and the consequences of incorrect diagnoses.

Our main analysis compared the flow monitor recorded at home with the PSG result from the sleep laboratory, though we also included data for the flow monitor recorded during the PSG night. We feel the main comparison to be the most appropriate one for three reasons. First, the analysis compares one device used in the home setting in which we intend to apply it with the PSG in the recommended laboratory setting. The analysis answers questions that might be posed by a sleep researcher needing to evaluate the extent to which the home-recorded flow monitor can be used in replacement of the in-laboratory PSG for measuring a study subject's degree of OSA. Second, the main analysis is more conservative, and although it includes error from the night-to-night variability in OSA measurement, it also includes potential error arising from the unattended use of the device in the field, including displacement of the nasal prongs and incorrect or noncompliant application of the device. Third, we see this type of comparison as a strength of our study, and this method of comparison has been recommended by consensus to assess how the test works in the setting in which it is intended to be applied (Flemons et al., 2003).

A possible limitation of this study may be the population from which the subjects were drawn. The subjects were selected from patients referred to a sleep clinic, not from a population of volunteers for behavioral research protocols. The prevalence of OSA in our study was high (79\%). This higher prevalence is not unexpected, given the subjects' high mean BMI and elevated levels of subjective sleepiness. It also reflects the referral pattern of the sleep center, where the subjects were first seen by a sleep physician, who requested the polysomnogram on the basis of clinical suspicion. Nevertheless, our inclusion criteria were broad. There was a wide spectrum of disease severity, as indicated by the range of apnea-hypopnea indices (Figure 2). There were also approximately equal numbers of subjects in the no-disease, mild-to-moderate, or severe OSA categories. The stability of the estimated test statistics will need to be confirmed by studies in different referral settings.

Table 2

Diagnostic Test Characteristics of the Flow Monitor (FW) Used at Home for Three Nights in Detecting Severe Obstructive Sleep Apnea

\begin{tabular}{ccccccc}
\hline $\begin{array}{c}\text { Threshold for } \\
\text { FW AHI }_{\mathbf{1 - 3}}\end{array}$ & Sensitivity & Specificity & $\begin{array}{c}\text { Positive } \\
\text { Likelihood Ratio }\end{array}$ & $\begin{array}{c}\text { Negative } \\
\text { Likelihood Ratio }\end{array}$ & $\begin{array}{c}\text { Positive } \\
\text { Predictive Value }\end{array}$ & $\begin{array}{c}\text { Negative } \\
\text { Predictive Value }\end{array}$ \\
\hline 59 & .18 & 1.00 & - & 0.82 & 1.00 & .69 \\
45 & .36 & .90 & 3.64 & 0.71 & .67 & .72 \\
$28^{*}$ & .91 & .75 & 3.64 & 0.12 & .67 & .94 \\
21 & 1.00 & .50 & 2.00 & 0.00 & .52 & 1.00 \\
\hline
\end{tabular}

Note-Sensitivity, specificity, positive likelihood ratio, negative likelihood ratio, positive predictive value, and negative predictive value for detecting polysomnogram apnea-hypopnea index $(\mathrm{AHI}) \geq 30$. "Point with the lowest distance from the upper left corner of the receiver operating characteristic curve and with the highest diagnostic odds ratio. 


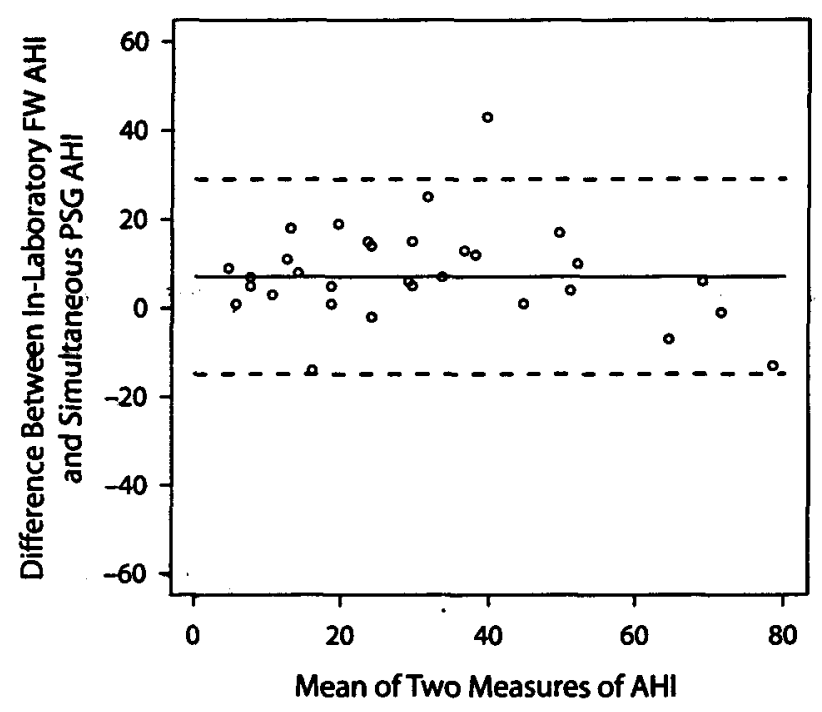

Figure 5. Bland and Altman (1986) plot comparing AHI from the in-laboratory PSG and the simultaneous flow monitor recording.

According to the area under the ROC curve, the questionnaire- and demographic-based MAPI was significantly less accurate than the flow monitor. The area under the curve of .68 was also lower than the value of .79 quoted in the original validation study for the instrument (Maislin et al., 1995). Logistic regression modeling showed the flow-monitor-derived AHI to be the best predictor of OSA, and the addition of the questionnaire predictive tool did not significantly contribute to the diagnosis. The referral pdttern and triaging on the basis of clinical features might also account for the performance of the questionnaire- and demographic-based instrument being poorer than that reported in the literature. However, this result also raises the issue of the importance of such simple objective tests, in contrast to questionnaire criteria for OSA identification, when used in research protocols. As well, given the frequency of insomnia symptoms in patients with OSA, and vice versa, it is important to have some simple and objective means to detect OSA in subjects being screened for insomnia-related research (Gooneratne et al., 2006).

The device used in this study also has potential utility in the diagnosis of sleep apnea, since it can be easily and cheaply distributed because of its size. Its ability to record for up to three nights may have the potential to reduce the effect of night-to-night variability in sleep apnea (Le Bon et al., 2000) and also absorb data loss from individual nights. Interestingly, in this study, restricting the analysis to the first night did not appear to compromise diagnostic accuracy.

There are several studies of diagnostic accuracy concerning portable flow monitors. In all cases, the devices were tested on a single night, and in the majority of cases, the devices were not studied in the home setting (Flemons et al., 2003; Ross et al., 2000). This may make diagnoses made on the basis of such devices vulnerable to night-tonight variability in OSA measurement (Mosko, Dickel, \& Ashurst, 1988). In addition, these studies do not fully reflect the setting in which the device would be used in practice. We are aware of only three studies looking at the use of portable recordings outside the laboratory setting. A study of 33 subjects evaluated a disposable thermistorbased flow monitor used at home: Valid data on this device were obtainable in only 17 subjects, and at a threshold AHI of 10 for the device and the polysomnogram, sensitivity was .52 and specificity .75 (Hollingworth, Tooby, Roberts, \& Hanning, 2003). Another study of 37 patients from a sleep center in which the same device was evaluated found sensitivity .55 and specificity of .70 for an AHI of 15 or greater (Pang et al., 2006). Another important issue is that thermistor-based single-channel devices may have limitations similar to those that have been demonstrated in polysomnographic studies comparing thermistor and nasal pressure methods of airflow detection (Berg, Haight, Yap, Hoffstein, \& Cole, 1997; Heitman, Atkar, Hajduk, Wanner, \& Flemons, 2002). Another study of 59 subjects used airflow information from one of two portable sleep systems in an ambulatory setting. Only $12 \%$ of the ambulatory recordings were performed at home, with the remaining subjects sleeping in a research center, and scoring was done manually by a scorer not blinded to the oximeter trace. With a threshold of 18 events/h for the device and the polysomnogram, sensitivity was .88 and specificity .92 (Ayappa, Norman, Suryadevara, \& Rapoport, 2004).

With the use of a single channel to detect nasal flow, it is plausible that the diagnostic accuracy of the device could be compromised in individuals with severe nasal occlusion. Subjects in this study were not excluded on the basis of nasal occlusion, and the degree of nasal patency or the presence of mouth-breathing was not assessed as part of the study. It is possible that this might affect a minority of patients, since the proportion of sleep spent exclusively mouth-breathing has been reported to be as low as $10 \%$ in a sleep center population, and in the same study individuals with more severe sleep apnea spent less time mouth-breathing (Oeverland, Akre, \& Skatvedt, 2002). The potential impact of nasal occlusion on the diagnostic accuracy of this device and the ability of the device to identify periods of significant mouth-breathing warrant further investigation, and meanwhile it would seem prudent to consider an alternative test in patients known to have severe nasal occlusion.

\section{CONCLUSIONS}

This evaluation of a flow-based single-channel monitor used at home has shown it to be a potentially useful tool in the identification of both high and low likelihood of the diagnosis of obstructive sleep apnea. With the use of appropriate thresholds, it can exclude the presence of disease with high sensitivity, and hence avoid the need for costly further investigations. The diagnostic device is portable and capable of recording data for up to three nights, and so will minimize error from night-to-night variability of the disease and accommodate data loss from nasal cannula displacement. This type of device may serve behavioral research requirements as a simple device for the economical determination of a subject's sleep-related breathing status. 


\section{AUTHOR NOTE}

The study was funded by DiagnoseIT and the CRC for Microtechnology, and K.K.H.W. was supported by the National Health Medical Research Council (NHMRC) Centre for Clinical Research Excellence in Respiratory and Sleep Medicine. R.R.G. is an NHMRC Practitioner Fellow. The funding sources placed no restrictions on the data analysis or publication of the study. A.R. (who was responsible for the data collection) and K.K.H.W. (who conducted the statistical analysis) declare that they have no competing interests. G.D. has provided paid consultative services to DiagnoseIT and to Respironics, Inc. G.U., J.A.H., and R.R.G. are scientific advisers to DiagnoselT. Correspondence concerning this article should be addressed to K. K. H. Wong, Woolcock Institute of Medical Research, P.O. Box M77, Missenden Road, Sydney, NSW 2050, Australia (e-mail: keithw@woolcock.org.au).

\section{REFERENCES}

American ACademy of Sleep Medicine Task Force (1999). Sleeprelated breathing disorders in adults: Recommendations for syndrome definition and measurement techniques in clinical research. The report of an American Academy of Sleep Medicine Task Force. Sleep, 22, 667-689.

Ayappa, I., Norman, R. G., Suryadevara, M., \& Rapoport, D. M. (2004). Comparison of limited monitoring using a nasal-cannula flow signal to full polysomnography in sleep-disordered breathing. Sleep, 27, 1171-1179.

Berg, S., Haight, J. S., Yap, V., Hoffstein, V., \& Cole, P. (1997) Comparison of direct and indirect measurements of respiratory airflow: Implications for hypopneas. Sleep, 20, 60-64.

BlAND, J. M., \& ALTMAN, D. G. (1986). Statistical methods for assessing agreement between two methods of clinical measurement. Lancet, 1, 307-310.

ENGLEMAN, H. M., \& JoFFE, D. (1999). Neuropsychological function in obstructive sleep apnoea. Sleep Medicine Reviews, 3, 59-78.

Flemons, W. W., LitTNer, M. R., Rowley, J. A., GAY, P., ANderson, W. M., HUDGEL, D. W., ET AL. (2003). Home diagnosis of sleep apnea: A systematic review of the literature. An evidence review cosponsored by the American Academy of Sleep Medicine, the American College of Chest Physicians, and the American Thoracic Society. Chest, 124, 1543-1579.

Gagnadoux, F., Pelletier-Fleury, N., Philippe, C., RakotonanaHARY, D., \& FLEURY, B. (2002). Home unattended vs. hospital telemonitored polysomnography in suspected obstructive sleep apnea syndrome: A randomized crossover trial. Chest, 121, 753-758.

Gooneratne, N. S., Gehrman, P. R., Nkwuo, J. E., Bellamy, S. L., Schutte-Rodin, S., Dinges, D. F., \& Pack, A. I. (2006). Consequences of comorbid insomnia symptoms and sleep-related breathing disorder in elderly subjects. Anchives of Internal Medicine, 166, 1732-1738.

Heitman, S. J., Atkar, R. S., Hajduk, E. A., Wanner, R. A., \& FlemONS, W. W. (2002). Validation of nasal pressure for the identification of apneas/hypopneas during sleep. American Journal of Respinatory \& Critical Care Medicine, 166, 386-391.
Hollingworth, L., Tooby, M., Roberts, D., \& Hanning, C. D. (2003). Practicality of the SleepStrip in postal screening for obstructive sleep apnoea. Journal of Sleep Research, 12, 157-159.

JAESCHKE, R., GUYATT, G. H., \& SACKETT, D. L. (1994). Users' guides to the medical literature: III. How to use an article about a diagnostic test: B. What are the results and will they help me in caring for my patients? The Evidence-Based Medicine Working Group. Journal of the American Medical Association, 271, 703-707.

JoHNS, M. W. (1991). A new method for measuring daytime sleepiness: The Epworth sleepiness scale. Sleep, 14, 540-545.

Le Bon, O., Hoffmann, G., Tecoo, J., Staner, L., Noseda, A., Pelc, I., \& LINKOwSKI, P. (2000). Mild to moderate sleep respiratory events: One negative night may not be enough. Chest, 118, 353-359.

Maislin, G., PAck, A. I., Kribis, N. B., Smith, P. L., SchWartz, A. R., KLINE, L. R., ET AL. (1995). A survey screen for prediction of apnea. Sleep, 18, 158-166.

Mosko, S. S., DickeL, M. J., \& AshuRst, J. (1988). Night-to-night variability in sleep apnea and sleep-related periodic leg movements in the elderly. Sleep, 11, 340-348.

Netzer, N. C., Stoohs, R. A., Netzer, C. M., Clark, K., \& Strohl, K. P. (1999). Using the Berlin Questionnaire to identify patients at risk for the sleep apnea syndrome. Annals of Internal Medicine, 131, 485-491.

OEVERLAND, B., AKRE, H., \& Skatvedt, O. (2002). Oral breathing in patients with sleep-related breathing disorders. Acta Oto-Laryngologica, 122, 651-654.

Pang, K. P., Dillard, T. A., Blanchard, A. R., Gourin, C. G., PODOLSKY, R., \& TERRIS, D. J. (2006). A comparison of polysomnography and the SleepStrip in the diagnosis of OSA. Otolaryngology: Head \& Neck Surgery, 135, 265-268.

Portier, F., Portmann, A., Czernichow, P., Vascaut, L., Devin, E., BENHAMOU, D., ET AL. (2000). Evaluation of home versus laboratory polysomnography in the diagnosis of sleep apnea syndrome. American Journal of Respiratory \& Critical Care Medicine, 162, 814-818.

RECHTSCHAFFEN, A., \& KALES, A. (EDS.) (1968). A manual of standardized terminology, techniques and scoring system for sleep stages of human subjects. Los Angeles: University of California.

Ross, S. D., Sheinhait, I. A., Harrison, K. J., KVasz, M., Connelly, J. E., SheA, S. A., \& Allen, I. E. (2000). Systematic review and metaanalysis of the literature regarding the diagnosis of sleep apnea. Sleep, 23, 519-532.

SLeEp Disorders AtLas Task Force of the American Sleer DisorDERS Association (1992). EEG arousals: Scoring rules and examples. A preliminary report from the Sleep Disorders Atlas Task Force of the American Sleep Disorders Association. Sleep, 15, 173-184.

Unger, G., Hedner, J. A., Grunstein, R. R., \& Williams, A. (2003). Diagnostic accuracy of a single channel nasal pressure recording in patients referred for investigation of sleep apnea. Sleep, 26, A401.

Young, T., Palta, M., Dempsey, J., Skatrud, J., Weber, S., \& Badr, S. (1993). The occurrence of sleep-disordered breathing among middleaged adults. New England Journal of Medicine, 328, 1230-1235.

(Manuscript received February 26, 2007; revision accepted for publication June 25,2007 .) 